\title{
A genetic game of trade, growth and externalities
}

\author{
Nedim M. Alemdar, Süheyla Özyıldırım* \\ Department of Economics, Bilkent University, 06533 Bilkent, Ankara, Turkey
}

Received 19 March 1997; accepted 20 August 1997

\begin{abstract}
A genetic algorithm is introduced to search for optimal policies in the presence of knowledge spillovers and local pollution in a dynamic North/South trade game. Noncooperative trade compounds inefficiencies stemming from externalities. Cooperative trade policies are efficient and yet not credible. Short of a joint maximization of the global welfare, transfer of knowledge remains as a viable route to improve world welfare. (C) 1998 Elsevier Science B.V. All rights reserved.
\end{abstract}

JEL classification: $\mathrm{C} 61 ; \mathrm{C} 73 ; \mathrm{F} 42$

Keywords: North/South dynamic trade game; Genetic algorithms; Externalities

\section{Introduction}

This paper aims to contribute to the recent literature exploring linkages between international trade, environmental degradation, and growth by bringing to the fore the dynamic gaming aspects of these issues. ${ }^{1}$ The framework we adopt is a dynamic trade game between North and South.

An extensive literature exists studying various aspects of the North/South trade. Galor (1986), an early precursor to this study, emphasizes the dynamic inefficiency of a noncooperative North/South trade wherein North is the engine of growth and South has a comparative advantage in resource production. Noncooperative resource pricing chokes up growth in the North. Labor market imperfections in the South, the surplus labor concomitant with positive

\footnotetext{
* Corresponding author. E-mail:suheyla@bilkent.edu.tr

${ }^{1}$ See among others Markusen (1975), Clemhout and Wan (1985), Levhari and Mirman (1980), and Dockner and Long (1993).
} 
subsistence wages, are the main culprit for the inefficiency. These market imperfections may, however, be a blessing in disguise if resource extraction pollutes the Southern environment; diminished Northern growth will check the pollution level in the South about which the North is indifferent.

In a series of papers, Chichilnisky $(1993,1994)$ draws attention to the salient feature of resource extraction in the South; (domestic) open access to Southern resources results in overexploitation and excessive pollution. Copeland and Taylor (1994) study the volume and the composition of trade between the North and South. Asymmetric endowment of human capital leads to trade between otherwise similar regions. Starting with a low level of human capital, South specializes in low-skill/pollution-intensive goods so that in the aftermath of trade, along with income the level of pollution rises. However, the long-run effects of trade on growth and pollution are unclear since accumulation of human capital is not allowed.

Neither Chichilnisky nor Copeland and Taylor consider the likely consequences of trade on knowledge spillovers. To the extent, trade also gives rise to knowledge spillovers, its undesirable impact on the pollution level will be mitigated. The beneficial effects of trade on capital accumulation or research and development are discussed by Grossman and Helpman (1991).

Our paper then addresses the issues related to trade and environment in a growth-cum-externalities setup. We intend to capture the impact of the Northern growth on the quality of the Southern environment in the presence of transboundary knowledge spillovers. Knowledge accumulation and spillovers bring about additional growth/pollution tradeoffs, and thus, help us identify sources of inefficiencies that have been hitherto overlooked.

The North/South trade in our model is specialized as in Galor (1986). In addition, resource extraction causes environmental pollution in the South. Also, knowledge accumulates in the North and diffuses costlessly to the South highlighting the public good nature of investment. We let the authorities in the South levy an export tax (or an import tariff) to internalize the local social cost of pollution and also to exploit their resource monopoly.

An additional contribution of the paper is in its methodology: We introduce a general purpose genetic algorithm (GA) to solve open-loop differential games of infinite duration. The lack of attention paid to the development of computational techniques to solve such problems was first addressed by Pau (1975a,b). We implement the algorithm $G A$ to solve numerically the North/South dynamic trade game.

In the GA search for optimal regional policies noncooperative and cooperative modes of behavior are considered. In the noncooperative Nash search, each region is represented by an artificially intelligent player, a $G A$, to adopt policies taking the rival's as given. Choices are evaluated in terms of their impact on the respective preferences (fitness functions) ignoring the side effects on the rival's fitness. Policies are then iteratively improved upon using a 
synchronous Darwinian search mechanism. Fittest policies are found if no improvement in 'lifetime' fitnesses is possible. South chooses resource prices with a view to maximize her own fitness. Resource prices also affect the rate of knowledge accumulation in the North, and hence, the Northern welfare which, however, does not enter into South's calculus of price determination. Likewise, in its search for optimal resource/knowledgc mix, the Northern intertemporal calculus discounts the fact that knowledge accumulation attenuates the detrimental side effects of the resource extraction to the Southern environment. As such, from the vantage point of global efficiency, noncooperative regional policies ultimaletly lead to underinvestment in knowledge capital.

In the cooperative search, the world fitness is represented as a weighted sum of each region's respective fitness. All externalities are thus internalized. Obviously, the resulting price and resource/knowledge paths are Pareto-efficient relative to a global fitness. It is worth noting here that though environmental pollution is local in nature, it has global ramifications calling for an international approach to appropriately internalize it.

Cooperative North/South trade agreements may fail to materialize for wellknown reasons such as the absence of enforcement mechanisms and high monitoring costs. Nevertheless, there is still scope to improve the world welfare even when regions adopt inefficient noncooperative policies. We show that 'enhancement' of knowledge diffusion has the potential to generate substantial welfare gains for both regions. For instance, if North, acting unilaterally, is to improve the Southern access to the stock of knowledge (related to the pollution abatement), this will improve not only the Southern, but via initially lower resource prices, North's own welfare as well.

The balance of the paper is organized as follows: Section 2 discusses the dynamic trade game between North and South. Section 3 introduces the Genetic Algorithm to solve open-loop dynamic Nash games. Section 4 contains numerical solution of the model and interpretations. A brief conclusion and further extensions follow in Section 5.

\section{The model}

\subsection{Non-cooperative North/South trade}

Consider a global economy comprised of two regions, namely, North and South. Employing a concave production technology $Y=F(K, R, u)$, North produces manufactured goods which are consumed and invested in the North or exported to the South at a fixed world price of unity. $K$ stands for broad capital measuring the current state of technical knowledge in the North (Griliches, 1979 ), $R$ is the raw material imported at a monopoly price determined by the South and $u$ captures all other uncounted determinants of output. 
The stock of knowledge accumulates in tandem with the rate of investment,

$$
\dot{K}_{t}=Y_{t}-p_{t} R_{t}-\delta K_{t}-C_{t}^{\mathcal{N}},
$$

where $p_{t}$ is the relative price of resources (Southern terms of trade), $0<\delta<1$ is the rate of depreciation of the broad capital. ${ }^{2}$ Henceforth, a dot over a variable denotes its time derivative while superscripts $\mathscr{N}$ and $\mathscr{S}$ stand for North and South, respectively. Eq. (1) indicates that the rate of knowledge accumulation will be set not only by the North's desired consumption profile, but also the South's. No investment takes place in the South so that the proceeds from the resource sale are totally consumed. Nonetheless, South indirectly affects the pace of knowledge accumulation in the North via its desired trajectory of terms of trade.

Northern optimal consumption plan maximizes the discounted Northern lifelong welfare, namely,

$$
\max _{C^{-N_{t}, R_{t}}} J_{\mathscr{N}}=\int_{0}^{\infty} \mathrm{e}^{-\rho_{\mathscr{N}} t} U\left(C_{t}^{\mathcal{N}}\right) \mathrm{d} t \quad 0<\rho_{\mathscr{N}}<1
$$

subject to Eq. (1) and $K(0)=K_{0}$ given, $C_{t}^{\mathcal{N}} \geq 0$ for all $t$. $\mathrm{U}\left(C_{t}^{\mathcal{N}}\right)$ is a strictly concave instantaneous utility function and $\rho_{\mathcal{H}}$ denotes the Northern time preference rate.

We assume endowment asymmetry and let the primary resource be produced only in the South by a constant returns to scale production function which is assumed, for simplicity, to be a fixed coefficient type. That is, $R_{t}=b L_{t}, b>0$ where $L_{t}$ is the labor employed at time $t^{3}$

Resource extraction causes pollution. Also knowledge, broad capital accumulated in the North, diffuses, albeit at a diminishing rate, to check the damage done to the Southern environment from resource extraction. Thus, patterns of trade and growth are further complicated by the presence of local and transboundary externalities. ${ }^{4}$

\footnotetext{
${ }^{2}$ Griliches (1979) discusses extensively various interpretation of depreciation in the context of broad capital.

${ }^{3}$ If it is assumed that the supply of labor in the South is perfectly elastic at a fixed real wage $w$ in terms of the manufacturing goods, the nature of the labor force coupled with the CRS production function would then determine labor income per unit of raw material as $w / b$. Competitive firms in the South will charge a price equal to the private marginal cost of resource extraction $w / b$. The assumed social planner in the South levies an export tax, not only to internalize the social cost of pollution, but also to extract monopoly profit from the North.

${ }^{4}$ In a dynamic game setup with transboundary pollution, Dockner and Long (1993) show that Pareto-efficient steady-state pollution level can be sustained with nonlinear Markov-Perfect strategies if discount rates are sufficiently low. Baç (1996) analyzes incentives to free-ride on transboundary pollution abatements when there are informational asymmetries.
} 
The type of pollution we consider has a high natural decay rate so that the cumulative effects are underplayed. With this specification the magnitude of the stock becomes proportional to the size of the flow defined as in Keeler et al. (1971), Markusen (1975). We specify the pollution $\mathscr{P}$ as

$$
\mathscr{P}=\frac{1}{\gamma} \frac{R_{t}^{\gamma}}{K_{t}^{\phi}}
$$

where $\gamma>1,0<\phi<1 . \gamma$ measures the exponential order of environmental damage due to extraction and $\phi$ is a knowledge diffusion (spillover) parameter, signifying the degree of applicability of knowledge to pollution reduction.

$\mathscr{P}$ enters into the Southern utility as a flow with a negative marginal utility. Given the Northern demand for resources, South chooses the terms of trade to maximize lifetime utility, i.e.,

$$
\max _{p_{t}} J_{\mathscr{S}}=\int_{0}^{\infty} \mathrm{e}^{-\rho_{\mathscr{S} t}} U\left(C_{t}^{\mathscr{S}}, \mathscr{P}_{t}\right) \mathrm{d} t \quad 0<\rho_{\mathscr{S}}<1
$$

subject to Eq. (1), (2) and

$$
\begin{aligned}
C_{t}^{\mathscr{S}} & =p_{t} R_{t}, \\
K(0) & =K_{0} \text { given, } C_{t}^{\mathscr{S}} \geq 0 \text { for all } t,
\end{aligned}
$$

where $\rho_{\mathscr{S}}$ is the Southern rate of time preference. Instantaneous utility is assumed separable in consumption $C_{t}^{\mathscr{P}}$, and pollution $\mathscr{P}_{t}$ so that $U\left(C_{t}^{\mathscr{S}}, \mathscr{P}_{t}\right)=U\left(C_{t}^{\mathscr{S}}\right)-D\left(\mathscr{P}_{t}\right) . U\left(C_{t}^{\mathscr{S}}\right)$ is strictly concave and $D\left(\mathscr{P}_{t}\right)$ is strictly increasing in $R_{t}$ and decreasing in $K_{r}$.

\subsection{Cooperative North/South trade}

In the design of cooperative strategies, the participants have to agree in advance upon how to distribute the potential gains from cooperation. The distributive outcome depends on the weights, $\omega$, that are put on the respective fitnesses. The determination of the value of $\omega$ most likely to prevail in a cooperative agreement requires a bargaining framework which recognizes the relative power of the participants. This is outside the scope of our inquiry. Instead, we consider an egalitarian allocation and assume exogenously given equal weights.

Let $\rho=\omega \rho_{\mathscr{N}}+(1-\omega) \rho_{\mathscr{S}}$ be the weighted time preference term. The Paretoefficient solution is found by

$$
\begin{gathered}
\max _{C \sim_{r}, R_{t}, P_{t}} J=\int_{0}^{\infty} \mathrm{e}^{-\rho t}\left\{\omega U\left(C_{t}^{\mathscr{V}}\right)+(1-\omega)\left[U\left(C_{t}^{\mathscr{S}}\right)-D\left(\mathscr{P}_{t}\right)\right]\right\} \mathrm{d} t \\
0<\rho<1
\end{gathered}
$$


s.t.

$$
\begin{aligned}
& \dot{K}_{t}=Y_{t}-p_{t} R_{t}-\delta K_{t}-C_{t}^{\mathcal{N}}, \\
& K(0)=K_{0} \text { given } C_{t}^{\mathcal{N}}, C_{t}^{\mathscr{S}} \geq 0
\end{aligned}
$$

Cooperation takes place on the premise that North and South can enter into binding commitments. Precommitment is difficult in the absence of suitable institutions which can enforce global decisions. Still, cooperative solutions, though lacking credibility, are important in so far as they establish an efficiency benchmark against which other solutions can be compared.

\section{Solution methods}

In the open-loop Nash solution of the game, each player faces a standard optimal control problem which is arrived at by fixing the other player's policies at some arbitrary functions. Hence, each such optimal control problem is parameterized in terms of some open-loop control policies which, however, do not alter the structure of the underlying optimization problems because of their open-loop character. Therefore, in principle, the necessary and/or sufficient conditions for open-loop Nash equilibria can be obtained by listing down the conditions required by each optimal control problem (via minimum principle) and then requiring that these all be satisfied simultaneously (Başar, 1986). Because of the couplings that exists between these various conditions, each one corresponding to the optimal control problem faced by one player, solving analytically for the Nash equilibria of our game poses a formidable task.

Recently, there has emerged a growing interest among economists in the computational aspects of complex dynamic structures which cannot be easily handled with traditional analytical methods. One search technique that has been successfully applied to such complex problems is the genetic algorithm. Genetic algorithm is a globally robust search mechanism which combines a Darwinian survival-of-the-fittest strategy to eliminate unfit characteristics and uses random information exchange, with exploitation of the knowledge contained in the previous solutions. Grefenstette (1986), Michalewicz (1992) and Krishnakumar and Goldberg (1992) used GA to optimize control problems with a single controller. Ǒzyldırım(1996) extended GA to solve open-loop difference games of finite horizon. In this paper we develop and implement $G A$ to solve open-loop differential games of infinite duration. Given the concave-convex structure of the model, a non $G A$ algorithm such as a gradient procedure could have performed equally well for numerical experimentation. However, since the application of $G A$ to differential games is quite new for the researchers, experimenting with such regular functional forms should be considered a start. Otherwise, the solution procedure is general and independent of the assumed 
functional forms. One aim of our paper is to propose it as a general purpose alternative game algorithm.

\subsection{Genetic algorithm}

Genetic algorithm initiated by Holland (1975) and further extended by De Jong is best viewed in terms of optimizing a sequential decision process involving uncertainty in the form of lack of a priori knowledge, noisy feedback and time varying payoff function. It is a highly parallel mathematical algorithm that transforms a set of (population) individual mathematical objects (typically fixed-length character strings patterned after chromosome strings), each with an associated fitness value, into a new population (i.e., the next generation) using operations patterned after Darwinian principles of reproduction and survival of the fittest after naturally occurring genetic operations (De Jong, 1993).

A $G A$ performs a multi-directional search by maintaining a population of individuals, $P(t)=\left\{x_{1}, \ldots, x_{n}\right\}$ where $x_{i}=\left\{x_{i 1}, \ldots, x_{i T}\right\}$; each individual, $x_{i}$ represents a potential solution vector to the problem at hand. An objective function (fitness) plays the role of an environment to discriminate between 'fit' and 'unfit' solutions. The population experiences a simulated evolution: at each generation the relatively 'fit' solutions reproduce while the relatively 'unfit' solutions die. During a single reproductive cycle fit individuals are selected to form a pool of candidates some of which undergo crossover and mutation in order to generate a new population.

Crossover combines the features of two parent chromosomes to form two similar offsprings by swapping corresponding segments of the parents. The intuition behind the applicability of the crossover operator is the information exchange between different potential solutions. Mutation arbitrarily alters one or more genes of a selected chromosome by a random change with a probability equal to the mutation rate pmut. The mutation operator introduces additional variability into the population. After some number of generations, the program converges. The best individuals represent the optimum solutions. ${ }^{5}$

\subsubsection{Genetic algorithm for noncooperative open-loop dynamic games}

Considering the fact that GA is a highly parallel mathematical algorithm, we offer a new solution procedure using GA to visualize situations or problems in which there are more than one performance measure and more than one intelligent controller (player) operating with or without coordination with others. We use both the optimization and the learning property of the $G A$ to solve the problems of multiple criteria optimization. Since the open-loop $n$ person Nash equilibria can be obtained as the joint solution to $n$ optimal control

\footnotetext{
${ }^{5}$ For further details, sec Goldberg (1989), Michalewicz (1992), and Arifovic (1994).
} 
problems (Başar and Oldser, 1982), then we can use $n$ parallel $G A s$ to optimize the control system.

In this setting, there are $n$ artificially intelligent players (controllers) who update their strategies through $G A$ and a referee, or a fictive player, who administers the parallel implementation of the algorithm and acts as an intermediary for the exchange of best responses. This fictive player (shared memory) has no decisive role but provides the best strategies in each iteration to the requested parties synchronously. In making his decisions, each player has certain expectations as to what the other players will do. These expectations are shaped through the information received from the shared memory in each iteration.

The following figure shows the general outline of the algorithm we use for the two-region dynamic trade game:

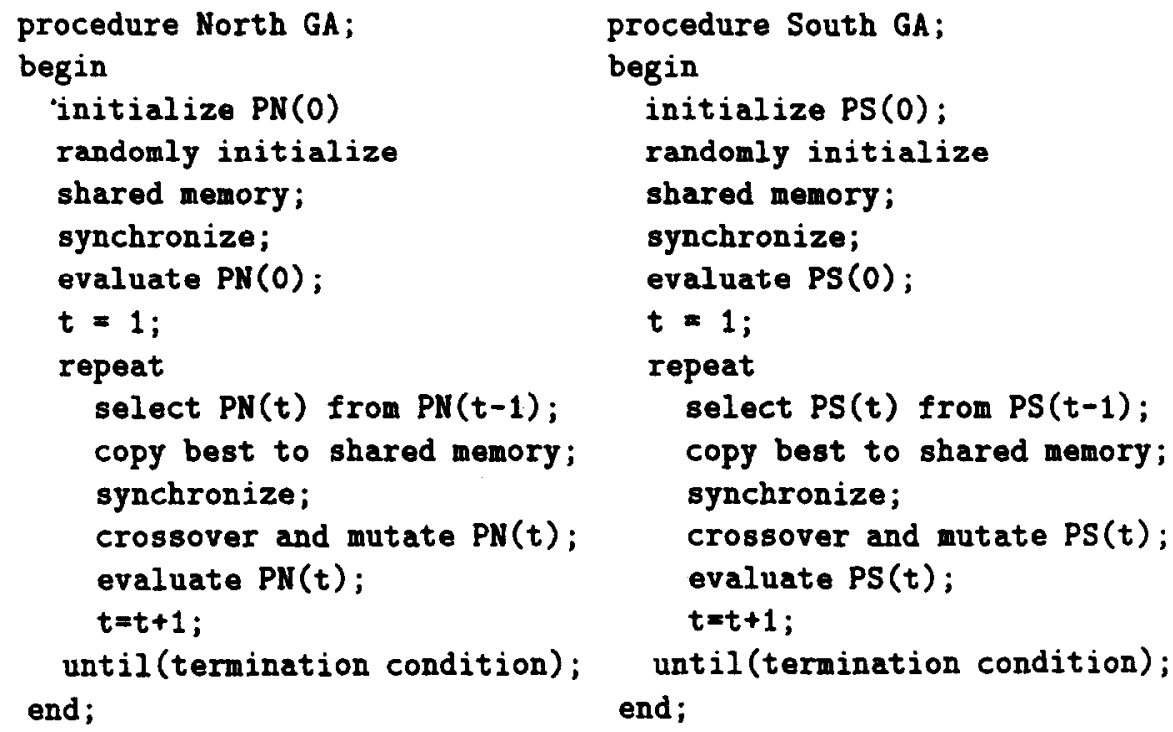

In the above algorithm, each side waits for the presence of the previous best structure of the other side in the synchronize statement.

In each step of this algorithm, two $G A s$ are solved. In order to reduce the time complexity, the two $G A s$ are solved for one generation while continuously sharing the best responses. This approach has the advantage that while reducing the time complexity it ensures that the convergence is to the global extremum.

\subsubsection{Genetic algorithm for cooperative games}

In a cooperative game, the strategic rivalry that exists in noncooperative games is eliminated via an 'arbitration' whereby the 'total fitness' as the weighted sum of each player's respective fitness is maximized. This is a typical 
control problem which can be solved by standard $G A$ techniques (Krishnakumar and Goldberg, 1992; Michalewicz, 1992).

In general, controls may involve constraints so that, either penalty functions or substitution may be used to transform the original problem to an unconstrained optimization problem for $G A$ implementation. ${ }^{6}$ For $n$ control variables, $T$ periods, and $k$ potential solutions, a $G A$ performs the following steps to optimize a control problem: (1) randomly generate an initial potential solution set, (2) evaluate the fitness value for a solution set of $n T k,(3)$ apply selection, crossover, and mutation operations to each set of solutions to reproduce a new population, (4) repeat steps (1)-(3) until computation is terminated according to a convergence criterion, (5) choose the solution set $n T$ based on the best fitness value from the current generations as the optimal solution set.

\section{Numerical experiments}

We need discrete reformulation of our model for numerical computation. Mercenier and Michel (1994) propose time aggregation to transform continuous-time infinite horizon optimal control problems into discrete-time approximations with the same steady state. This approach imposes consistency constraints on the joint formulation of preferences and accumulation equations. It is shown that this consistency is achieved by a simple restriction on the choice of discount factor. In the appendix we show that their results extend to openloop dynamic Nash games. Then we exploit the inherent parallelism in $G A$ to solve the time-aggregated North/South dynamic trade game.

The discrete-time approximation of infinite horizon North/South trade model with steady-state invariance is as follows: ${ }^{7}$

$$
\begin{aligned}
& \max J^{i}=\sum_{m=0}^{M-1} \theta_{m}^{i} \Delta_{m} U^{i}\left(t_{m}\right)+\theta_{M-1}^{i} G^{i}\left(K\left(t_{M}\right)\right) \\
& \text { s.t. } K\left(t_{m+1}\right)-K\left(t_{m}\right)=\Delta_{m}\left[Y\left(t_{m}\right)-p\left(t_{m}\right) R\left(t_{m}\right)-C^{\mathcal{N}}\left(t_{m}\right)-\delta K\left(t_{m}\right)\right], \\
& K\left(t_{0}\right)=K_{0} \text { given, } C^{i} \geq 0 i=\mathscr{N}, \mathscr{S}
\end{aligned}
$$

where $M$ is the assumed terminal time when the stationary state is reached, $\Delta_{m}$ a scalar factor that converts the continuous flow into stock increments,

\footnotetext{
${ }^{6}$ We have linear constraints both as equalities and inequalities. The equalities are eliminated at the start by substitution. The constrained problem is then transformed to an unconstrained problem by associating penaltics with all constraint violations which are included in the fitness functions. We used arbitrarily large negative numbers to penalize constraint violations. See Michalewicz (1992) for various $G A$ approaches to handle linear constraints.

${ }^{7}$ See appendix for derivation.
} 
$\Delta_{m}=t_{m+1}-t_{m}$ and $\theta_{m}^{i}$ the sequence of discount factors of the region $i$ for which the stationary solution of the discrete-time problem is equivalent to the corresponding continuous-time problem. These sequences are given by the following recursions:

$$
\theta_{m}^{\mathscr{N}}=\frac{\theta_{m-1}^{\mathcal{N}}}{1+\rho_{\mathscr{N}} \Delta_{m}}, \theta_{0}^{\mathscr{N}}>0 \text { and } \theta_{m}^{\mathscr{S}}=\frac{\theta_{m-1}^{\mathscr{S}}}{1+\rho_{\mathscr{S}} \Delta_{m}}, \theta_{0}^{\mathscr{S}}>0
$$

The functions $G^{i}(\cdot)$ denote the terminal values.

For numerical experiments, we adopt the following particular functional forms:

$$
U\left(C_{t}^{i}\right)= \begin{cases}\frac{C_{t}^{i^{1-\sigma}}}{1-\sigma} & \text { for } \sigma>0, \quad \sigma \neq 1, \\ \log C_{t}^{i} & \text { for } \sigma=1,\end{cases}
$$

and

$$
D\left(\mathscr{P}_{\imath}\right)=\frac{d}{\gamma} \frac{R_{t}^{\gamma}}{K_{t}^{\phi}}, \quad d>0
$$

where $d$ converts pollution to utility. Also,

$$
Y_{t}=a K_{t}^{\alpha} R_{t}^{\beta}, \quad \alpha+\beta<1 \text { and } a>0 .
$$

All uncounted inputs $u$, are normalized to one for simplicity. The following set of parameter values are assumed:

$$
\begin{array}{ll}
\alpha=0.80, & \beta=0.15, \quad \gamma=2, \quad a=1, \quad b=1, \quad d=0.00001 \\
\sigma=1.50, \quad \delta=0.08, \quad \rho_{\mathcal{N}}=0.02, \quad \rho_{\mathscr{S}}=0.02, \quad \omega=0.50, \quad \phi=0.15 .
\end{array}
$$

These parameter values are assumed for the purposes of illustration, however, they are not totally unjustified. Similar values of $\alpha, \sigma, \delta, \rho_{i}$ and $a$ are used by Auerbach and Kotlikoff (1987) in a different context. $d$ is so chosen to conform with the assumed utility function. $\phi$ parameterizes the importance of the effects of knowledge spillovers in the North/South trade game. To highlight the significance of the knowledge spillover, we run the experiment with $\phi=0.30$ as well. $\beta, \gamma$, and $b$ are chosen to satisfy parameter restrictions and are inconsequential to our arguments about knowledge spillovers. ${ }^{8}$

\footnotetext{
${ }^{8}$ The genetic operators in this paper were done using the public domain GENESIS package (Grefenstette, 1990) on a SUN SPAC-1000 running Solaris 2.4. A typical run uses population size, $j=50$, runs 15 million generations for noncooperative game and 30 million generations for cooperative game, crossover rate is 0.60 and mutation rate is 0.03 . None of the results depends on the values of genetic operators other than run time by the choice of number of generations. For each parameter set, we have to implement three separate GAs. Hence, we are limited by the increased computational costs in our scope for a complete sensitivity analysis.
} 
In the time-aggregated model, we assume 21 periods $(M=20)$ with a dense equally spaced gridding of the time horizon $T(t(M)=200)$, which is sufficient to capture the convergence over time.

As mentioned earlier, we simultaneously run two separate genetic programs, $G A^{\mathscr{N}}$ and $G A^{\mathscr{S}}$, to solve the noncooperative game. $G A^{\mathscr{N}}$ generates a population of candidate solutions (chromosomes), $K(t)$ representing the Northern accumulated knowledge. $G A^{\mathscr{S}}$ produces the population of chromosomes $p(t)$ denoting the set of Southern price strategies. Structures $K_{j}, p_{j}$ in each population $(j=1,2, \ldots, 50)$ are represented as binary strings $\left(\left\{\begin{array}{ll}0 & 1\end{array}\right\}\right)$ of length $l$. For string $j$ of length $l(=10)$, decoding works as follows:

$$
K_{j}(t)=\sum_{h=1}^{l} a_{j}^{h}(t) 2^{h-1}, \quad p_{j}(t)=\sum_{h=1}^{l} a_{j}^{h}(t) 2^{h-1},
$$

where $a_{j}^{h}(t)$ is the value $\left\{\begin{array}{ll}0 & 1\end{array}\right\}$ taken at the $h$ th position in the string. After strings are decoded, integers $K_{j}(t)$ and $p_{j}(t)$ are normalized in order to obtain a real number value.

Since $K_{0}$ is given and $p_{0}$ is free, in each iteration (generation), $G A^{\mathscr{N}}$ computes $M$ while $G A^{\mathscr{S}}$ finds $M+1$ structures each with a domain, $D_{i}=[\underline{d}, d] \subseteq \Re ; i=p, K . D_{i}$ is cut into $(\bar{d}-\underline{d}) 2^{10}$ equal size ranges. Thus, the noncooperative game has the minimal search domain of $2^{410}=$ 2.64423E + 123 .

Cooperative solutions are computationally much more complex than noncooperative ones. In the latter case, the search for the optimum consists of two one-dimensional problems, while the former represents one two-dimensional problem. In the cooperative experiment, three chromosomes, $p_{t}, K_{t}$ and $R_{t},(62$ structure) are searched in the minimal domain of $2^{620}$.

Regional decisions are updated using genetic operators, selection, crossover, and mutation. The selection strategy is elitist so that the best performing strategy in the population of survivors is retained. This selection rule is a natural candidate in noncooperative Nash games. Therefore, it is especially crucial for the dynamic noncooperative game algorithm as it requires best responses be mutually exchanged. Were it not for the elitist selection, the best structures may disappear making for a nonconvergence.

Since $G A s$ work with constant-size populations of candidate solutions, $G A$ searches are initialized from a number of points. Initialization routines may vary. We, however, start from randomly generated populations so as not to prejudice the convergence of the populations on the initial ones. Therefore, a randomly initialized $G A$ is less prone to numerical instability that may be caused by initialization. For the $G A$ parameters which might cause instability, we used the parameters chosen and studied on various optimization experiments by Grefenstette (1986). From the result of the experiments in the paper, the convergence is self-evident. 
The termination conditions are specified beforehand as a certain number of iterations. We gradually increase the number of iterations until no further improvements are observed.

\subsection{The results}

Fig. 1 and Table 1 summarize our numerical findings based on the assumed parameter values. First, from Table 1 note that North/South cooperation generates considerable welfare improvements for both regions. Moreover, South has more to gain from such a regime switch indicating the severity of the Northern noncooperation.

Also to be observed from Table 1 is the increase in regional welfares attendant with stronger knowledge diffusion. More significantly, comparing the welfares under the noncooperative regime with augmented knowledge dissemination $(\phi=0.30)$ and cooperation with restricted knowledge spillover $(\phi=0.15)$, we see substantial gains materialize even with uncoordinated trading policies attesting to the importance of access to knowledge.

The policy implication is that even if parties fail, say due to enforcement problems, to realize the first best solution, they may still achieve significant improvements in global welfare by strengthening the knowledge flows from North to South. It may be costly to setup global institutions to monitor and enforce North/South cooperations. To the extent that knowledge diffusion can be enhanced relatively cheaply, regions may opt to cooperate on sharing knowledge related to pollution control.

Studying Fig. 1 number of results stand out. To wit, in the long-run cooperation yields sizable increases in knowledge stock, resource use, resource/ knowledge mix, pollution level, and consumption irrespective of the extent of knowledge spillovers. Southern terms of trade first deteriorates to recover later on. Furthermore, this recovery is faster with the greater degree of spillovers so that in the long-run coordinated resource prices ultimately surpass the noncooperative ones.

Along the cooperative path Southern terms of trade equate the marginal social benefits of resource use (the marginal utility of manufactured goods times the marginal product of resources) to the marginal pollution costs in the South. Without cooperation, Southern terms of trade depreciates at the margin if the welfare improvement due to the increased marginal export revenue plus the marginal benefit from the accelerated knowledge accumulation in the North (valued at the shadow price of knowledge in the South which reflects also the positive knowledge externality) is greater than the increase in the marginal pollution cost (in terms of Southern disutility).

As such, Southern noncooperation adds to the dynamic inefficiency to the extent her market power limits knowledge growth in the North. This deleterious effect of resource monopoly, however, is mitigated to the degree South 

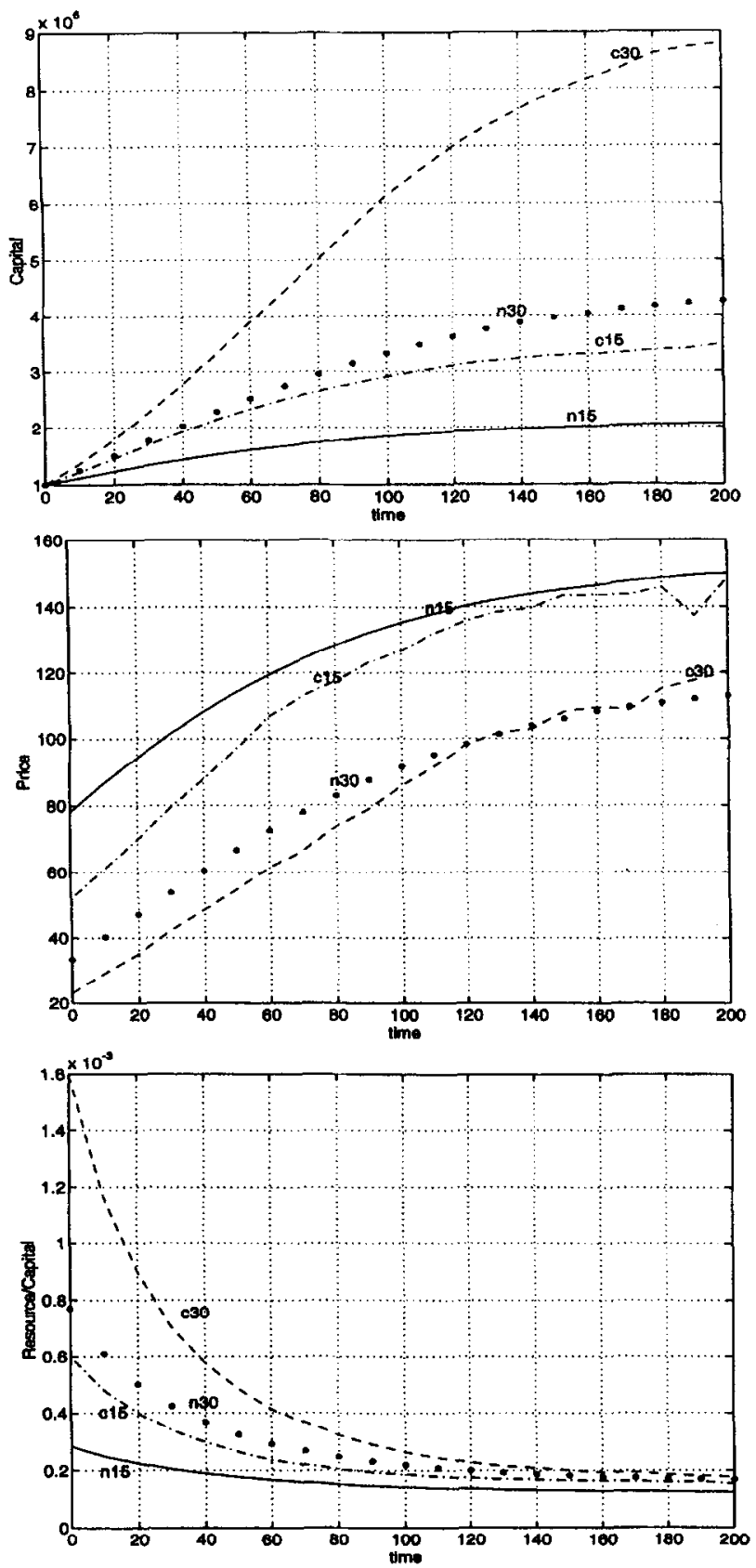

Fig. 1. $\mathrm{n} 15$ (c15) stands for noncooperation (cooperation) with $\phi=0.15$. Likewise for $\phi=0.30$. 

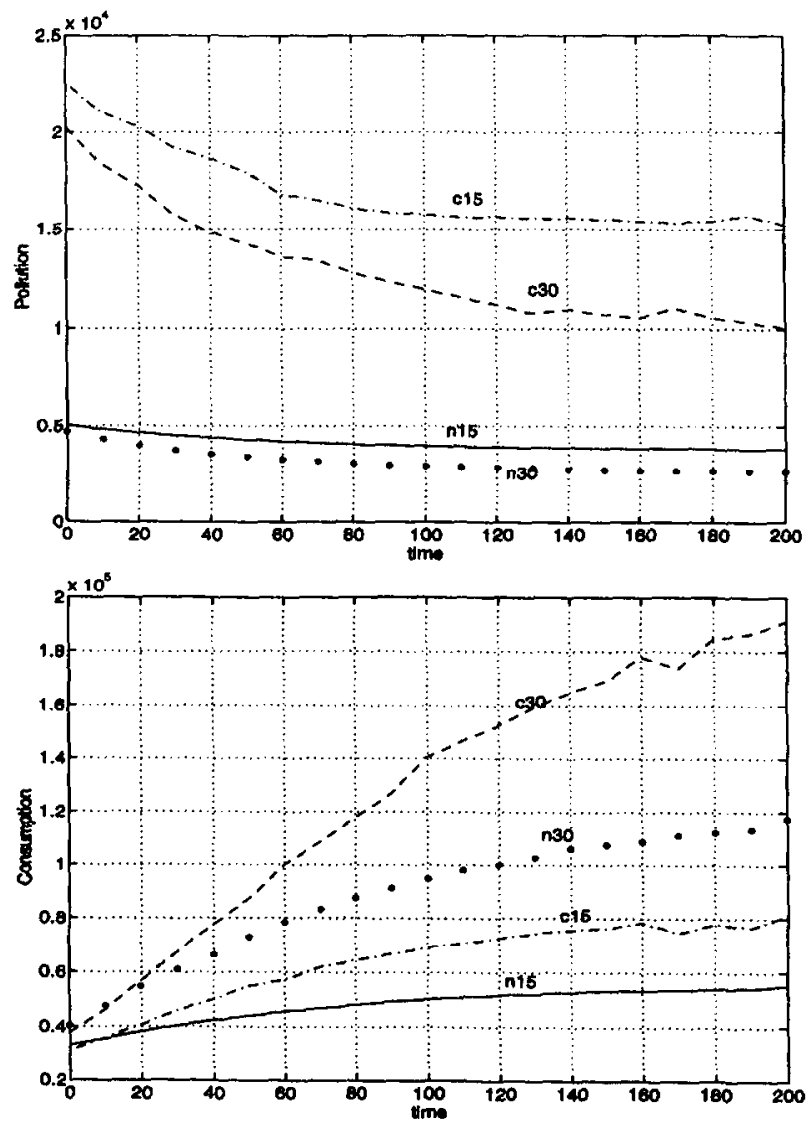

Fig. 1. (Continued).

Table 1

The total discounted welfares

\begin{tabular}{llllll}
\hline $\begin{array}{l}\text { Rate of } \\
\text { diffusion }(\phi)\end{array}$ & North & & \multicolumn{2}{l}{ South } & \\
\cline { 2 - 3 } & Noncooperative & Cooperative & & Noncooperative & Cooperative \\
\hline 0.15 & -0.058138 & -0.054725 & & -0.072729 & -0.065882 \\
0.30 & -0.047346 & -0.045009 & & -0.060418 & -0.054318 \\
\hline
\end{tabular}

Note: Because of the assumed utility function, values closer to zero indicate higher welfare.

internalizes the knowledge spillovers. On the other hand, noncooperative Northern investment plans are globally inefficient as they understate the true world marginal benefit by the amount of the marginal improvement in the Southern welfare due to the incremental reduction in the pollution level. 
Therefore, major gains from cooperation accrue initially when the knowledge stock is so low that a rapid investment plan is called for. Southern terms of trade obliges by shifting down and tilting towards future to accommodate a faster adjustment. Consequently, the knowledge stock accumulates at a more rapid rate; the pollution level starts higher but falls off precipitously; and the regional consumptions rise more swiftly.

Another important set of results has to do with the long-run effects of an increase in the knowledge diffusion parameter $\phi$. First, note the rise in the optimal long-run resource/capital ratio. This will be true because, a higher rate of knowledge diffusion will reduce the long-run pollution cost and thereby the supply price of resources, and make the increased use of resources for any given level of knowledge optimal. Also, worthy of notice is the increase in the stationary knowledge stock and the fall in the pollution level. The marginal reduction in the pollution level due to a higher $K$ outweighs the incremental increase due to a higher $R$ so that the overall long-run pollution will fall.

For the dynamic inefficiency of the noncooperative trade regime and the failure of cooperation, we provide the following explanations: In the noncooperative mode the shadow value (the marginal benefit) of the knowledge stock differs for the two regions as the regions have different preferences (fitnesses) leading to conflicting policies and harmful 'policy externalities'. Moreover, when policies are chosen with a view to maximize own fitnesses taking the rival's as given, the 'incentive' effects of the policies are ignored. The South chooses resource prices for any 'given investment policy' of the North, thus, ignoring the fact that a lower price today (lower consumption) may 'induce' the North to invest more today which then leads to higher prices (higher Southern consumption) as the higher knowledge stock shifts the demand for resources tomorrow. The North, on the other hand, ignores the fact that an initially higher investment profile (lower consumption) may induce South to ask for lower resource prices today in return for higher prices tomorrow (as the demand for resources will shift) and also to higher Northern consumption in the future as the amount to be invested will be lower in the future (higher Northern consumption).

Parties ignore the incentive effects for the fact that promises are not credible. If South were to offer cooperative prices, it would not be optimal for the North to invest as much promised as along the cooperative path: North will consume more and invest less. Likewise, if North were to commit itself to the investment plan along the cooperative path, then it would not be optimal for the South to ask for the cooperative prices: South will raise prices and consume more. Failing to cooperate, the parties will revert to their respective Nash strategies. 


\section{Concluding remarks}

This paper has introduced genetic algorithms to search for optimal policies in the presence of knowledge spillovers and local pollution in a dynamic North/South trade game. Cooperative trade policies are efficient but fail to be enforceable. Noncooperative trade policies compound inefficiencies stemming from externalities. Competitive resource production in the South overpollutes whereas 'local' internalization of pollution together with resource monopoly limit growth and trade and result in underpollution.

Because of the spillovers, the stock of knowledge is partially a common property (see Grossman and Helpman, 1991 for this point). The North underinvests because it cannot fully capture the benefits from investment in knowledge. Even though the pollution is local in the South, the North still has an incentive to speed up knowledge diffusion. The South in turn internalizes the benefits from accelerated spillovers in the form of reduced pollution costs, compensating North with initially lower resource prices.

The model can be extended in number of directions. For instance, one obvious modification would be to allow pollution to accumulate which then adds an extra dimension to the intertemporal pollution/growth tradeoff. The transboundary effects of pollution can be considered to further add to the dynamic gaming aspects of international relations. These, however, would come at the expense of increased computational cost as there would be an additional state variable in the system dynamics.

Also, other forms of noncooperative behavior, such as Stackelberg leader/ follower setup, could be considered. In this framework one needs to utilize the necessary conditions from the follower's problems as constraints to the leader. In the $G A$ game algorithm we develop, it is not obvious how to handle this without having to first analytically derive the necessary conditions for the follower. This, however, would violate the integrity of the GA as a "blind" algorithm. In order to numerically solve Stackelberg leader/follower model, a new $G A$ game algorithm needs to be devised.

\section{Appendix A.}

\section{A.1. A general sketch of GA for the solution of dynamic games}

Two parallel GAs use genetic operators to iterate on constant-size populations, $P i(t), i=\mathscr{N}, \mathscr{S}$ of candidate solutions. During each iteration step, $t$, called a generation, structures in the current populations are evaluated to reproduce 
new populations as

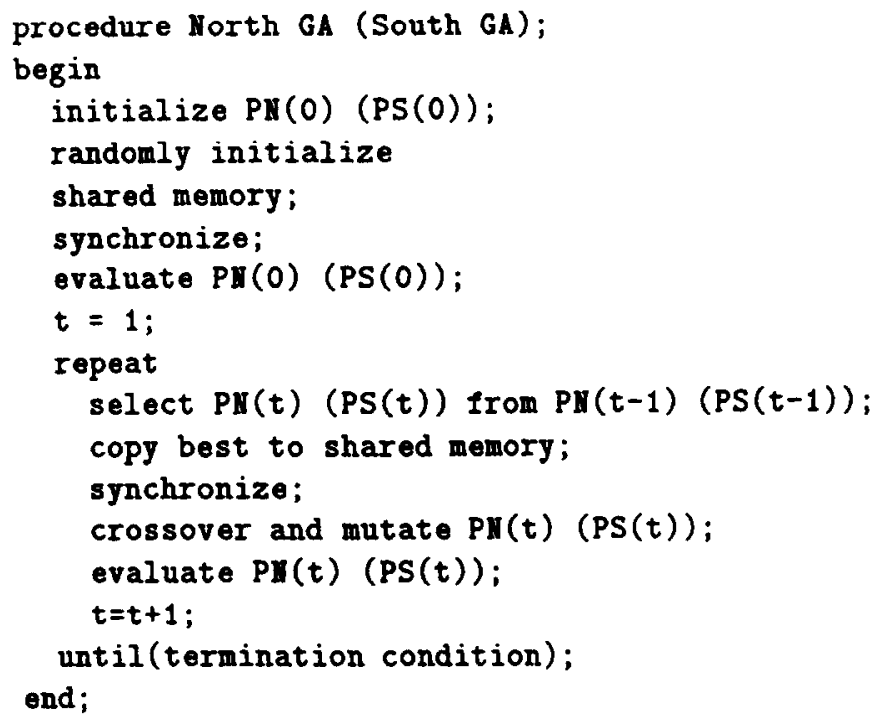

The initial populations are randomly produced and a randomly selected individual from each population is sent to the computer shared memory to be exchanged synchronously. As both GAs (North and South) need to reach the shared memory, a priority protocol is required. By synchronization, one $G A$ uses the memory if the memory is not currently in use by the other. If the memory is in use, however, the late arriver waits to access the memory. The whole procedure to reach the shared memory is the synchronization process. Upon the exchange of the information, the initial populations $P N(0)(P S(0)$ are evaluated. At $t=1$, a new population, $P i(t)$, is formed from the previous, $P i(t-1)$. We select populations to reproduce on the basis of their relative fitnesses. Best performing individuals in each population are sent (copied) to the shared memory again to be exchanged synchronously. The selected individuals are then recombined using genetic operators, crossover and mutation to form new populations. Crossover is the most important genetic operator. It operates by swapping corresponding segments of a string of parents to produce offsprings. For example, if parents are represented by vectors, $x_{1}=\left(a_{1}, b_{1}, c_{1}\right.$, $\left.d_{1}, e_{1}\right)$ and $x_{2}=\left(a_{2}, b_{2}, c_{2}, d_{2}, e_{2}\right)$, then crossing the vectors from the second to fifth elements would produce the offsprings $\left(a_{1}, b_{1}, c_{2}, d_{2}, e_{2}\right)$ and $\left(a_{2}, b_{2}, c_{1}, d_{1}, e_{1}\right)$. The mutation operator arbitrarily alters one or more components of a selected structure in order to introduce variability in the populations so that the likelihood of getting stuck at a local extremum is reduced. This procedure of creating new populations, exchange of the best individuals and 
evaluation of the populations in each generation iterate a fixed number of times or until $G A s$ find an acceptable approximate solution.

\section{A.2. Discrete-time approximation of the model with steady-state invariance}

We generalize the result by Mercenier and Michel (1994) to transform continuous-time infinite horizon control problems to discrete-time approximations for multi-player games. Consider an $n$-player continuous-time dynamic game with the state vector $x(t) \in \mathfrak{R}^{k}$ and the control vector $u_{i} \in \mathfrak{R}^{v_{i}}, i=1,2, \ldots, n$ :

$$
\max J^{i}=\int_{0}^{\infty} \mathrm{e}^{-\rho_{i} t} g_{i}\left(x(t), u_{1}(t), \ldots, u_{n}(t)\right) \mathrm{d} t
$$

s.t. $\quad \dot{x}(t)=f\left(x(t), u_{1}(t), \ldots, u_{n}(t)\right), \quad x(0)=x_{0}$ given.

The following relations for $i=1,2 \ldots, n$ characterize the stationary open-loop Nash equilibria $\left(\hat{x}, \hat{u}_{1}, \ldots, \hat{u}_{n}, \hat{q}_{1}, \ldots, \hat{q}_{n}\right)$ :

$$
\begin{aligned}
& f\left(\hat{x}, \hat{u}_{1}, \ldots, \hat{u}_{n}\right) \\
& \quad=0, \rho_{i} \hat{q}_{i}=\nabla_{x} H^{i}\left(\hat{x}, \hat{u}_{1}, \ldots, \hat{u}_{n}\right) \text {, and } \nabla_{u} H^{i}\left(\hat{x}, \hat{u}_{1}, \ldots, \hat{u}_{n}\right)=0,
\end{aligned}
$$

where $H^{i}\left(x, u_{1} \ldots, u_{n}\right)=g_{i}\left(x, u_{1} \ldots, u_{n}\right)+q_{i}^{\prime} f\left(x, u_{1}, \ldots, u_{n}\right)$ is the current valued Hamiltonian, $q_{i}(t)^{\prime} \in \Re^{k}$ is the transpose of the costate vector.

The discrete-time approximation of the above problem is

$$
\begin{aligned}
& \max \tilde{J}^{i}=\sum_{m=0}^{\infty} \theta_{m}^{i} \Delta_{m} g_{i}\left(x\left(t_{m}\right), u_{1}\left(t_{m}\right), \ldots, u_{n}\left(t_{m}\right)\right) \\
& \text { s.t. } x\left(t_{m+1}\right)-x\left(t_{m}\right)=\Delta_{m} f\left(x\left(t_{m}\right), u_{1}\left(t_{m}\right), \ldots, u_{n}\left(t_{m}\right)\right), \quad x\left(t_{0}\right)=x_{0} \text { given, }
\end{aligned}
$$

where $\Delta_{m}$ converts the continuous flows into stock increments, i.e., $\left(\Delta_{m}=\right.$ $t_{m+1}-t_{m}$ ) and $\theta_{m}^{i}$ is the sequence of discount factors for which the stationary solution of the discrete-time problem is the same that of the continuous-time problem. The recurrence for $\theta_{m}^{i}$ is generated from the optimality conditions of the discretized game.

The optimality conditions satisfy

$$
\begin{aligned}
& \Delta_{m} \nabla_{u_{i}}\left\{g_{i}\left(x\left(t_{m}\right), u_{1}\left(t_{m}\right), \ldots, u_{n}\left(t_{m}\right)\right)+q_{i}\left(t_{m}\right)^{\prime} f\left(x\left(t_{m}\right), u_{1}\left(t_{m}\right), \ldots, u_{n}\left(t_{m}\right)\right)\right\}=0, \\
& \theta_{m}^{i} \Delta_{m}\left\{\nabla _ { x } \left\{g_{i}\left(x\left(t_{m}\right), u_{1}\left(t_{m}\right), \ldots, u_{n}\left(t_{m}\right)\right)\right.\right. \\
& \left.\left.\quad+q_{i}\left(t_{m}\right)^{\prime} f\left(x\left(t_{m}\right), u_{1}\left(t_{m}\right), \ldots, u_{n}\left(t_{m}\right)\right)\right\}\right\}-\theta_{m-1}^{i} q_{i}\left(t_{m-1}\right)+\theta_{m}^{i} q_{i}\left(t_{m}\right)=0 .
\end{aligned}
$$

Imposing the stationary equivalence of the continuous and discrete-time problems, using Eq. (A.1) in Eq. (A.2) and Eq. (A.3), the following recursions are 
obtained:

$$
\theta_{m-1}^{i} \hat{q}_{i}=\theta_{m}^{i} \hat{q}_{i}+\theta_{m}^{i} \Delta_{m} \rho_{i} \hat{q}_{i}
$$

for any $\theta_{0}^{i}>0$.

For the $G A$ application, we truncate the original infinite horizon continuoustime problem. The finite horizon discrete-time approximation becomes

$$
\max \widetilde{J}=\sum_{m=0}^{M-1} \theta_{m}^{i} \Delta_{m} g_{i}\left(x\left(t_{m}\right), u_{1}\left(t_{m}\right), \ldots, u_{n}\left(t_{m}\right)\right)+\beta_{M}^{i} G^{i}\left(x\left(t_{M}\right)\right) \quad i=1, \ldots, n
$$

s.t. $x\left(t_{m+1}\right)-x\left(t_{m}\right)=A_{m} f\left(x\left(t_{m}\right), u_{1}\left(t_{m}\right), \ldots, u_{n}\left(t_{m}\right)\right), \quad x_{0}$ given,

where it is assumed that stationary solution is reached at $T_{M}$.

The steady-state invariance property imposes specific restrictions on the choice of functions $G^{i}(\cdot)$. The terminal value $G^{i}(\cdot)$ is

$$
G^{i}(\hat{x})=\int_{0}^{\infty} \mathrm{e}^{-\rho_{i} t} g_{i}\left(\hat{x}, \hat{u}_{1}(x), \ldots, \hat{u}_{n}(x)\right) \mathrm{d} t=\frac{1}{\rho_{i}} g_{i}\left(\hat{x}, \hat{u}_{1}(x), \ldots, \hat{u}_{n}(x)\right),
$$

so that recursion is terminated at $\beta_{M}^{i}=\theta_{M-1}^{i}$.

\section{A.3. Tables}

Table 2

Noncooperative game with $\phi=0.15$

\begin{tabular}{llllllllll}
\hline$t$ & $K_{t}$ & $p_{t}$ & $R_{t}$ & $\mathscr{P}_{t}$ & $C_{t}^{r}$ & $C_{t}^{\mathscr{l}}$ & $R_{t} / K_{t}$ & $\mathscr{P}_{d} / K_{t}$ \\
\hline 0 & 1000000.000 & 77.967 & 283.125 & 5045.758 & 33064.556 & 22074.356 & 0.000283 & 0.005046 \\
1 & 1120234.604 & 86.442 & 279.039 & 4818.407 & 35627.665 & 24120.597 & 0.000249 & 0.004301 \\
2 & 1234604.106 & 94.252 & 276.186 & 4652.048 & 38184.480 & 26031.175 & 0.000224 & 0.003768 \\
3 & 1340175.953 & 101.564 & 273.253 & 4498.064 & 40373.921 & 27752.721 & 0.000204 & 0.003356 \\
4 & 1436950.147 & 108.045 & 271.306 & 4388.060 & 42354.554 & 29313.215 & 0.000189 & 0.003054 \\
5 & 1524926.686 & 114.027 & 269.286 & 4284.599 & 44088.316 & 30705.942 & 0.000177 & 0.002810 \\
6 & 1604105.572 & 119.345 & 267.680 & 4201.621 & 45662.203 & 31946.254 & 0.000167 & 0.002619 \\
7 & 1674486.804 & 124.164 & 266.037 & 4123.555 & 46772.060 & 33032.227 & 0.000159 & 0.002463 \\
8 & 1739002.933 & 128.485 & 264.800 & 4062.196 & 48103.356 & 34022.724 & 0.000152 & 0.002336 \\
9 & 1794721.408 & 132.141 & 263.921 & 4016.228 & 49353.218 & 34874.649 & 0.000147 & 0.002238 \\
10 & 1841642.229 & 135.298 & 263.001 & 3972.867 & 50203.064 & 35583.532 & 0.000143 & 0.002157 \\
11 & 1882697.947 & 137.957 & 262.438 & 3942.815 & 51027.299 & 36205.093 & 0.000139 & 0.002094 \\
12 & 1917888.563 & 140.283 & 261.849 & 3914.248 & 51790.614 & 36733.103 & 0.000137 & 0.002041 \\
13 & 1947214.076 & 142.278 & 261.241 & 3887.233 & 52206.763 & 37168.797 & 0.000134 & 0.001996 \\
14 & 1973607.038 & 143.939 & 260.983 & 3871.732 & 52931.005 & 37565.710 & 0.000132 & 0.001962 \\
15 & 1994134.897 & 145.269 & 260.702 & 3857.408 & 53316.818 & 37871.848 & 0.000131 & 0.001934 \\
16 & 2011730.205 & 146.432 & 260.411 & 3843.733 & 53386.591 & 38132.566 & 0.000129 & 0.001911 \\
17 & 2029325.513 & 147.595 & 260.122 & 3830.200 & 53746.547 & 38392.741 & 0.000128 & 0.001887 \\
18 & 2043988.270 & 148.592 & 259.824 & 3817.304 & 54085.868 & 38607.874 & 0.000127 & 0.001868 \\
19 & 2055718.475 & 149.423 & 259.519 & 3805.080 & 54405.853 & 38778.193 & 0.000126 & 0.001851 \\
20 & 2064516.129 & 149.922 & 259.545 & 3803.405 & 55337.091 & 38911.488 & 0.000126 & 0.001842 \\
\hline & & & & & & & & \\
\hline
\end{tabular}


Table 3

Cooperative game with $\phi=0.15$

\begin{tabular}{llllllllll}
\hline$t$ & $K_{t}$ & $p_{t}$ & $R_{t}$ & $\mathscr{P}_{t}$ & $C_{t}{ }^{\prime}$ & $C_{t}^{\prime f}$ & $R_{t} / K_{t}$ & $\mathscr{P}_{t} / K_{t}$ \\
\hline 0 & 1000000.000 & 51.818 & 598.651 & 22558.876 & 31053.778 & 31021.008 & 0.000599 & 0.022559 \\
1 & 1225806.448 & 60.909 & 586.921 & 21031.282 & 35933.968 & 35748.814 & 0.000479 & 0.017157 \\
2 & 1460410.552 & 69.853 & 584.233 & 20298.802 & 40712.731 & 40810.621 & 0.000400 & 0.013899 \\
3 & 1697947.216 & 79.384 & 574.457 & 19186.562 & 45916.300 & 45602.827 & 0.000338 & 0.011300 \\
4 & 1923753.664 & 87.889 & 571.281 & 18622.863 & 50150.338 & 50209.026 & 0.000297 & 0.009680 \\
5 & 2140762.468 & 96.979 & 564.927 & 17921.275 & 54982.562 & 54786.292 & 0.000264 & 0.008371 \\
6 & 2331378.304 & 106.657 & 550.020 & 16771.980 & 57228.799 & 58663.375 & 0.000236 & 0.007194 \\
7 & 2507331.376 & 112.815 & 548.553 & 16501.593 & 61906.551 & 61885.174 & 0.000219 & 0.006581 \\
8 & 2653958.944 & 117.361 & 543.666 & 16071.253 & 64431.200 & 63804.988 & 0.000205 & 0.006056 \\
9 & 2791788.856 & 123.079 & 541.711 & 15835.157 & 66743.358 & 66673.302 & 0.000194 & 0.005672 \\
10 & 2912023.456 & 126.745 & 542.199 & 15763.725 & 69246.953 & 68720.993 & 0.000186 & 0.005413 \\
11 & 3017595.304 & 132.023 & 540.733 & 15595.052 & 70757.598 & 71389.460 & 0.000179 & 0.005168 \\
12 & 3105571.852 & 135.689 & 542.444 & 15626.375 & 72579.693 & 73603.737 & 0.000175 & 0.005032 \\
13 & 3175953.076 & 138.622 & 541.711 & 15531.866 & 74402.654 & 75092.852 & 0.000171 & 0.004890 \\
14 & 3228739.000 & 139.648 & 542.688 & 15549.477 & 75613.083 & 75785.369 & 0.000168 & 0.004816 \\
15 & 3275659.828 & 143.460 & 542.199 & 15487.926 & 76374.164 & 77784.150 & 0.000166 & 0.004728 \\
16 & 3304985.332 & 143.607 & 541.222 & 15411.513 & 78440.272 & 77723.273 & 0.000164 & 0.004663 \\
17 & 3319648.096 & 143.607 & 540.000 & 15331.820 & 74690.809 & 77547.801 & 0.000163 & 0.004619 \\
18 & 3375366.568 & 145.806 & 541.711 & 15390.638 & 77901.150 & 78984.908 & 0.000160 & 0.004560 \\
19 & 3398826.976 & 137.302 & 547.087 & 15681.350 & 76759.806 & 75116.168 & 0.000161 & 0.004614 \\
20 & 3483870.964 & 148.739 & 540.000 & 15221.176 & 81028.038 & 80319.062 & 0.000155 & 0.004369 \\
\hline
\end{tabular}

Table 4

Noncooperative game with $\phi=0.30$

\begin{tabular}{llllllllll}
\hline$t$ & $K_{t}$ & $p_{t}$ & $R_{t}$ & $\mathscr{P}_{t}$ & $C_{t}^{r}$ & $C_{t}^{Y}$ & $R_{t} / K_{t}$ & $\mathscr{P}_{t} / K_{t}$ \\
\hline 0 & 1000000.000 & 33.343 & 769.100 & 4687.433 & 40390.275 & 25644.170 & 0.000769 & 0.004687 \\
1 & 1249266.862 & 40.205 & 760.908 & 4291.783 & 47658.693 & 30592.521 & 0.000609 & 0.003435 \\
2 & 1506842.620 & 47.067 & 754.121 & 3985.032 & 54830.827 & 35494.555 & 0.000500 & 0.002645 \\
3 & 1764418.377 & 53.803 & 747.500 & 3734.328 & 60987.681 & 40217.422 & 0.000424 & 0.002116 \\
4 & 2021994.135 & 60.283 & 743.355 & 3545.101 & 66417.730 & 44812.030 & 0.000368 & 0.001753 \\
5 & 2279569.892 & 66.637 & 739.617 & 3385.541 & 72826.629 & 49286.121 & 0.000324 & 0.001485 \\
6 & 2520527.859 & 72.610 & 734.888 & 3243.138 & 78298.376 & 53360.227 & 0.000292 & 0.001287 \\
7 & 2744868.035 & 77.947 & 732.542 & 3141.080 & 83202.928 & 57099.637 & 0.000267 & 0.001144 \\
8 & 2952590.420 & 82.903 & 729.719 & 3049.443 & 87493.390 & 60496.074 & 0.000247 & 0.001033 \\
9 & 3143695.015 & 87.478 & 726.686 & 2967.777 & 91280.228 & 63569.031 & 0.000231 & 0.000944 \\
10 & 3318181.818 & 91.417 & 725.973 & 2914.347 & 94835.891 & 66366.589 & 0.000219 & 0.000878 \\
11 & 3476050.831 & 95.103 & 723.980 & 2858.231 & 97954.462 & 68852.409 & 0.000208 & 0.000822 \\
12 & 3617302.053 & 98.280 & 723.134 & 2817.681 & 100047.483 & 71069.273 & 0.000200 & 0.000779 \\
13 & 3750244.379 & 101.329 & 721.700 & 2776.297 & 102748.345 & 73129.473 & 0.000192 & 0.000740 \\
14 & 3866568.915 & 103.998 & 720.379 & 2740.904 & 106069.849 & 74917.967 & 0.000186 & 0.000709 \\
15 & 3957966.764 & 106.031 & 719.810 & 2717.463 & 107546.961 & 76322.329 & 0.000182 & 0.000687
\end{tabular}


Table 4

Continued

\begin{tabular}{lllllllll}
\hline$t$ & $K_{\mathrm{t}}$ & $P_{\mathrm{t}}$ & $R_{\mathrm{t}}$ & $\mathscr{P}_{t}$ & $C_{\mathrm{t}}{ }^{\prime}$ & $C_{t}^{\varphi^{f}}$ & $R_{t} / K_{t}$ & $\mathscr{P}_{t} / K_{\mathrm{t}}$ \\
\hline 16 & 4041055.718 & 108.192 & 716.810 & 2678.120 & 108703.692 & 77552.851 & 0.000177 & 0.000663 \\
17 & 4115835.777 & 109.589 & 718.356 & 2674.931 & 111021.151 & 78724.278 & 0.000175 & 0.000650 \\
18 & 4173998.045 & 110.860 & 718.100 & 2661.795 & 112211.082 & 79608.752 & 0.000172 & 0.000638 \\
19 & 4223851.417 & 112.004 & 717.454 & 2647.559 & 113297.510 & 80357.660 & 0.000170 & 0.000627 \\
20 & 4265395.894 & 113.021 & 716.437 & 2632.319 & 117610.283 & 80972.110 & 0.000168 & 0.000617 \\
\hline
\end{tabular}

Table 5

Cooperative game with $\phi=0.30$

\begin{tabular}{|c|c|c|c|c|c|c|c|c|}
\hline 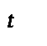 & $K_{t}$ & $p_{i}$ & $R_{t}$ & $\mathscr{P}_{\mathrm{r}}$ & $C_{t}^{t}$ & $C_{t}^{\psi}$ & $R_{t} / K_{t}$ & $P_{t} / K_{1}$ \\
\hline 0 & 1000000.000 & 23.072 & 1597.290 & 20217.970 & & & & \\
\hline 1 & 136 & & & & & & & \\
\hline 2 & & & & & & & & \\
\hline 3 & & & & & & & & \\
\hline 4 & 2766351.580 & 48.686 & & & & & & \\
\hline 5 & 3304057.180 & & & & & & & \\
\hline 6 & & & & & & & & \\
\hline 7 & & & & & & & & \\
\hline 8 & & & & & & & & \\
\hline 9 & 55694 & & & & & & & \\
\hline 10 & & & & & & & & \\
\hline 11 & 65654 & & & & & & & \\
\hline 12 & 69797 & & & & & & & \\
\hline 13 & 733 & & & & & & & \\
\hline 14 & 7640822.743 & 102.933 & & & & & & \\
\hline 15 & & & & & & & & \\
\hline 16 & 8178528.343 & 109.532 & 1581.602 & 10552.658 & 178158.237 & 173236.347 & 0.000193 & 0.001290 \\
\hline 17 & 8354800.761 & 108.637 & 1622.392 & 11033.182 & 173812.724 & 176252.449 & 0.000194 & 0.001321 \\
\hline 18 & 8619248.717 & 115.125 & 1595.049 & 10565.188 & 184793.166 & 183629.538 & 0.000185 & 0.001226 \\
\hline 19 & 8725043.631 & 117.585 & 1581.378 & 10346.920 & 186627.283 & 185946.965 & & \\
\hline 20 & 8795560.464 & 120.270 & 1561.206 & 10060.310 & 191094.069 & 187765.933 & 0.000177 & 0.001144 \\
\hline
\end{tabular}

\section{References}

Arifovic, J., 1994. Genetic algorithm learning and the cobweb model. Journal of Economic Dynamics and Control 18, 3-28.

Auerbach, A.L., Kotlikoff, L.J., 1987. Dynamic Fiscal Policy, Cambridge University Press, Cambridge, New York.

Baç, M., 1996. Incomplete information and incentives to free ride on international environmental resources. Journal of Environmental Economics and Management 30, 301-315. 
Başar, T., Oldser, G.J., 1982. Dynamic Noncooperative Game Theory. Academic Press, New York.

Başar, T., 1986. A tutorial on dynamic and differential games. in: Başar, T. (ed.), Dynamic Games and Applications in Economics. Springer, Berlin.

Chichilnisky, G., 1993. North-South trade and the dynamics of renewable resources. Structural Change and Economic Dynamics 4, 219-248.

Chichilnisky, G., 1994. North, South trade and the global environment. American Economic Review $84,851-874$.

Clemhout, S., Wan Jr., H., 1985. Dynamic common property resource and environmental problems. Journal of Optimization Theory and Applications 46, 471-481.

Copeland, B.R., Taylor, M.S., 1994. North-South trade and environment. Quarterly Journal of Economics 109, 755-787.

De Jong, K.A., 1993. Genetic algorithms are NOT function optimizers. in: Whitley, L.D. (Ed.), Foundations of Genetic Algorithms 2. Morgan Kaufmann, San Mateo, CA.

Dockner, E.J., Long, N.V., 1993. International pollution control: cooperative versus noncooperative strategies. Journal of Environmental Economics and Management 24, 13-29.

Galor, O., 1986. Global dynamic inefficiency in the absence of international policy coordination: a North-South case. Journal of International Economics 21, 137-149.

Goldberg, D.E., 1989. Genetic Algorithms in Search, Optimization and Machine Learning, Addison Wesley, Reading, MA.

Grefenstette, J.J., 1986. Optimization of control parameters for genetic algorithms. IEEE Transactions on Systems, Man, and Cybernetics 16, 122-128.

Grefenstette, J.J., 1990. A user's guide to GENESIS Version 5.0, manuscript.

Griliches, Z., 1979. Issues in assessing the contribution of R\&D to productivity growth. The Bell Journal of Economics, 10, 92-116.

Grossman, G.M., Helpman, E., 1991. Trade, innovation and growth. American Economic Review Papers and Proceedings 80, 71-86.

Holland, J.H., 1975. Adaptation in Natural and Artificial Systems. Ann Arbor, University of Michigan Press.

Keeler, E., Spence, M., Zeckhauser, R., 1971. The optimal control of pollution. Journal of Economic Theory 4, 19-34.

Krishnakumar, K., Goldberg, D.E., 1992. Control system optimization using genetic algorithm. Journal of Guidance, Control, and Dynamics 15, 735-738.

Levhari, D., Mirman, L., 1980. The great fishwar: an example using a dynamic Cournot-Nash solution. Bell. Journal 11, 322-334.

Markusen, J.R., 1975. Cooperative control of international pollution and common property resources. Quarterly. Journal of Economics 89, 618-632.

Mercenier, J., Michel, P., 1994. Discrete-time finite horizon approximation of infinite horizon optimization problems with steady-state invariance. Econometrica 62, 635-656.

Michalewicz, Z., 1992. Genetic Algorithm + Data Structures = Evolution Program. Springer, Berlin.

Özy1ldınm, S., 1996. Three-country trade relations: A discrete dynamic game approach. Computers and Mathematics with Applications 32, 43-56.

Pau, L.F., 1975a. Differential games and a Nash equilibrium searching algorithm. SIAM Journal of Control 13, 835-842.

Pau, L.F., 1975b. A differential game among sectors in a macroeconomy. Automatica 11, 473-485. 